\title{
Caracterización sociodemográfica del cuidador familiar de la persona mayor en una localidad de Bogotá-Colombia
} Socio-demographic Characteristics of the Caregiver for Elderly People in a Location in Bogota-Colombia

\section{Caracterização sociodemográfica do cuidador familiar da pessoa idosa numa região da cidade de Bogotá-Colômbia}

\author{
Blanca Nieves Piratoba-Hernández, Enf., Esp., MSc.* \\ Jeimy Nathaly Rozo-Gutiérrez, Enf., MSc. (c). **
}

\begin{abstract}
Resumen
Introducción: El incremento de la población adulta mayor y de las enfermedades crónicas no trasmisibles aumenta la demanda de cuidados permanentes que recaen en las mujeres. El reconocimiento de las características sociodemográficas permite identificar la situación que viven los cuidadores. Objetivo: Caracterizar los cuidadores familiares de la persona mayor en situación de dependencia, para contribuir al desarrollo de programas adaptables a las necesidades de los cuidadores. Metodología: Diseño descriptivo cuantitativo de corte transversal entre agosto de 2010 a marzo 2011. La recolección de información mediante entrevista domiciliaria, con cuestionario de caracterización socio demográfica. La muestra intencional de 91 cuidadores. Para el análisis estadístico se empleó programa SPSS versión 15. Resultados: Se evidenciaron cuidadores familiares en un rango de edad de 36 a 59 años (53\%), la mayoría mujeres $81 \%$, con niveles de escolaridad de primaria o bachillerato incompleto $45 \%$, casados(as) $36 \%$, con dedicación completa al hogar $53 \%$,afiliados al régimen subsidiado o del Estado 87\%; el rol de cuidador familiar lo
\end{abstract}

han ejercido por más de 37 meses $(87 \%)$, con una dedicación diaria de 24 horas (54\%) y la mayoría es cuidado por el hijo(a). Estos cuidadores no poseen herramientas suficientes para enfrentar las situaciones negativas derivadas del cuidado de la persona mayor. Conclusiones: Características como la edad, nivel de educación, tiempo de dedicación al cuidado, la red de apoyo, la ocupación y la situación socioeconómica hacen inminente la búsqueda de alternativas para los cuidadores que no cuentan con recursos para acceder a niveles óptimos de información; estos resultados permiten a diferentes sectores ejecutar acciones, generar programas de acuerdo con necesidades identificadas que impacten sobre la calidad de vida de los cuidadores; la normativa existente del Sistema General de Seguridad Social en Salud ofrece apoyo mínimo a los cuidadores. [Piratoba-Hernández BN, Rozo-Gutiérrez JN. Caracterización sociodemográfica del cuidador familiar de la persona mayor en una localidad de Bogotá-Colombia. MedUNAB 2015; $18(1): 51-57]$

Palabras claves: Cuidadores; Enfermería; Factores Socioeconómicos; Enfermedad Crónica; Dependencia (Psicología).

\footnotetext{
* Enfermera, Especialista en Administración de Servicios de Salud, Magister en Enfermería con Énfasis en Cuidado al Paciente Crónico, Profesora Auxiliar Universidad Nacional de Colombia- Sede Bogotá, Facultad de Enfermería-Departamento de Enfermería. Grupo de Investigación Cuidado Cultural de la Salud.

** Enfermera, Magister en Salud Pública, Asociación Colombiana de Facultades de Enfermería, ACOFAEN, Bogotá- Colombia.
}

Correspondencia: Blanca Nieves Piratoba Hernández. Universidad Nacional de Colombia -Sede Bogotá, Facultad de Enfermería- Departamento de Enfermería Grupo de Investigación Cuidado Cultural de la Salud. Carrera 30 No 45-03-.Tel: (57-1)3165000 -Extensión 17082-Bogotá-Colombia. E.mail: bnpiratobah@unal.edu.co. 


\section{Abstract}

Introduction: A rise in the elderly population and also in the Chronic Non-Communicable Diseases (NCD) has increased the demand of women providing permanent care. The recognition of socio-demographic characteristics allows to identify the situation caregivers go through. Objective: To identify special characteristics of family caregivers of dependent elderly people in order to contribute to the development of programs that provide assistance. Method: A quantitative cross sectional descriptive design from August 2010 to March 2011. Data collection was done through a face to face interview using the sociodemographic characterization survey. There was a practical sample with 91 caregivers. The SPSS version 15 statistics program was used to analyze the data. Results: It was learned that family caregivers' age ranged from 36 to 59 , and that $81 \%$ of them were women who attended only elementary school or did not finish high school $45 \%$. $36 \%$ of them were married, $53 \%$ of them stayed home, and $87 \%$ of them had health insurance. Most of the caregivers were children taking care of parents who have done this job 24/7 for more than 37 months (87\%). These caregivers do not have enough tools to face negative situations that could result when taking care of elderly people. Conclusions: Characteristics such as age, education level, time dedicated to the care, support network, occupation, and socioeconomic status make necessary the search for alternatives for caregivers who do not have resources to access to optimal levels of information. These results allow different sectors to implement actions and generate programs according to the needs that impact on the quality of life for caregivers. The existing regulations of the General Health System provide minimal support for caregivers. [Piratoba-Hernández BN, Rozo-Gutiérrez JN. Sociodemographic characteristics of the caregiver for elderly people in a location in Bogota-Colombia. MedUNAB 2015; 18(1): 51-57].

Keywords: Caregivers; Nursing; Socioeconomic Factors; Chronic Disease; Dependency (Pshychology).

\section{Introducción}

A nivel internacional la pirámide poblacional muestra tendencia hacia el incremento de la población adulta mayor, como consecuencia del control de la natalidad, y de variaciones de los estilos de vida, aumento de la longevidad, introducción de tecnologías en salud y múltiples cambios de los sistemas de salud del mundo $(1,2)$.

Estas dinámicas son expuestas en los informes sanitarios mundiales como agentes atribuibles de la incidencia y prevalencia de las enfermedades crónicas no transmisibles, las cuales registran en los últimos años un aumento desmesurado, siendo las causales del $80 \%$ de las muertes en los países, en especial de aquellos con ingresos medios y bajos (3-5), cifras que sobrepasan la capacidad tecnológica y del recurso humano disponible $(6,7)$.

\section{Resumo}

Introdução: O aumento da população idosa e das doenças crônicas não transmissíveis tem aumentado a procura de cuidados permanentes realizados por mulheres. O reconhecimento das características sociodemográficas permite identificar a situação vivida pelas cuidadoras. Objetivo: Caracterizar os familiares cuidadores das pessoas idosas em situação de dependência, para ajudar a desenvolver programas adaptáveis às necessidades das pessoas cuidadoras. Metodologia: Elaboração de um projeto quantitativo, descritivo e transversal de agosto de 2010 a março de 2011. A informação foi adquirida através de entrevistas domiciliares e das respostas ao questionário de caracterização sociodemográfica. A amostra intencional foi de 91 cuidadores. Para a análise estatística utilizou-se o programa SPSS em sua versão 15. Resultados: Foi evidente a faixa etária das pessoas familiares cuidadoras entre os 36 e 59 anos (53\%); na sua maioria são mulheres (81\%), com níveis de escolaridade fundamental ou ensino médio incompleto $(45 \%)$, casados (36\%), com total dedicação ao lar (53\%), afiliadas ao seguro social subsidiado ou do Estado (87\%). O trabalho de familiares cuidadores tem sido exercido por mais de 37 meses (87\%); com uma dedicação diária de 24 horas (54\%) e a maioria é cuidado pelo filho(a). Estes cuidadores não têm os conhecimentos suficientes para lidar com situações negativas decorrentes da assistência às pessoas idosas. Conclusões: Características como idade, nível de escolaridade, tempo dedicado ao cuidado, rede de apoio, ocupação e status socioeconômico fazem urgente a procura de alternativas para os cuidadores que não têm os recursos para obter níveis ótimos de informação; estes resultados permitem que diferentes setores possam implementar ações e criar programas de acordo com as necessidades identificadas, que possam impactar na qualidade de vida das pessoas cuidadoras. As regras existentes do Sistema Geral de Segurança Social em Saúde fornece suporte mínimo para as cuidadoras. [Piratoba-Hernández BN, RozoGutiérrez JN. Caracterização sociodemográfica do cuidador familiar da pessoa idosa numa região da cidade de BogotáColômbia. MedUNAB 2015; 18 (1): 51-57].

Palavras-chave: Cuidadores; Enfermagem; Fatores Socioeconômicos; Doença Crônica; Dependência (Psicologia).

Las enfermedades crónicas son prevenibles bajo estrategias viables y costo-efectivas basadas en el manejo de los factores de riesgo, sin embargo su escaso impacto sobre los indicadores de salud han demarcado un reto enorme para reducir las consecuencias sobre la calidad de vida de las poblaciones, porque invade esferas familiares con transformaciones en las dinámicas interpersonales y físicas que aceleran la pérdida de autonomía para la realización de actividades de la vida diaria, requiriendo así de actores cercanos que acompañen la aceptación, afrontamiento y resolución de la patología. Varios estudios reportan que la presencia de dichos actores produce mayor adhesión al tratamiento, control de la enfermedad y estrecha la probabilidad de complicaciones a mediano y largo plazo (3-6).

En América Latina y el Caribe el panorama demográfico es similar al internacional con respecto al aumento de la 
población mayor $(6,8,9)$; a su vez se ilustra un similar predominio de las enfermedades crónicas no transmisibles como productores de vulnerabilidad y dependencia funcional $(1,2,8,9-12)$, la dependencia es traducida a la dificultad de realizar las actividades de la vida diaria tanto en términos de acción como de pensamiento, por lo que requiere apoyo del otro; lo que trae consigo un aumento de la demanda de cuidados permanentes que obliga la disponibilidad de un cuidador dentro de la red inmediata que posee la persona mayor (1, 6-9).

Los estudios nacionales e internacionales de características sociodemográficas de los cuidadores familiares han reportado que en su mayoría son mujeres las que asumen la responsabilidad de brindar ayuda y apoyo de forma voluntaria y sin remuneración (13); con acciones de cuidado físico, social, psicológico y religioso; y un elevado compromiso afectivo, durante las 24 horas del día $(9,10$, 12,14-16).

Las cuidadoras han sido ubicadas en edades entre los 36 a los 59 años, etapa denominada Intermedia por la variedad de funciones que realizan $(11,12,17)$, son únicas cuidadoras (8), y llevan más de 18 meses en este rol, con una dedicación diaria de 13 a 24 horas (11), pertenecientes al estrato socioeconómico $2(8)$, y $3(15,18)$. El reconocimiento de las características ha permitido identificar la dinámica situacional en la que viven los cuidadores, a mayor pérdida de funcionalidad de la persona con enfermedad crónica, más sobrecarga percibirá el cuidador; comprometiendo su salud física y mental, lo que disminuye la percepción de bienestar y confort $(13,16,19)$.

Esta transición en el mundo ha permitido construir un panorama de las Enfermedades Crónicas No Trasmisibles ECNT e involucrar a múltiples disciplinas, como la Enfermería que ha sido una de las profesiones de la salud vinculada a los procesos educativos e investigativos para el monitoreo, vigilancia y seguimiento de las enfermedades de larga duración (9); la valoración de las características sociodemográficas, contribuye a generar estrategias y desarrollar programas de apoyo a cuidadores que den respuesta a sus necesidades $(12,16)$. El objetivo de este estudio se orientó a la caracterización de los cuidadores familiares de la persona mayor con Enfermedad Crónica no Transmisible en situación de dependencia.

\section{Metodología}

El estudio tiene un diseño descriptivo cuantitativo de corte transversal desarrollado entre agosto de 2010 y marzo de 2011. La muestra fue de tipo intencional de 91 cuidadores de personas mayores en situación de dependencia vinculados al Proyecto 496 "años dorados", de la Localidad de Usaquén en Bogotá-Colombia. Los criterios de inclusión que se tuvieron en cuenta fueron: cuidadores familiares de las personas mayores vinculados al proyecto
496, mayores de edad, que aceptaran participar en el estudio voluntariamente; los criterios de exclusión fueron: tener diagnósticos de trastornos mentales y/o cognitivos. La recolección de información fue realizada mediante visita domiciliaria, se empleó el cuestionario de caracterización socio-demográfico elaborado por el Grupo de Cuidado al Paciente Crónico de la Facultad de Enfermería de la Universidad Nacional de Colombia. Para el análisis estadístico de los datos fue empleado el programa SPSS versión 15 , se hizo cálculo de medidas de frecuencia absolutas y relativas de cada una de las variables sociodemográficas; se cumplió el control de confiabilidad de la base de datos.

Los aspectos éticos en la investigación cumplieron con la normativa para Colombia: el criterio del respeto a su dignidad y la protección de sus derechos y su bienestar a través del consentimiento informado, de acuerdo con La Ley 911 de 2004, para la profesión de Enfermería (20), para Colombia la resolución 8430 de 1993 (21), constituida bajo la Declaración De Helsinki (equipo de profesionales idóneos, rigurosidad científica, protección a la dignidad humana y a la confidencialidad).

El estudio recibió el aval institucional del comité de ética y de la Subdirección de la Secretaría de Integración Social Usaquén, Bogotá D.C-Colombia, para la realización de la investigación. Se obtuvo la aprobación del uso de la encuesta de caracterización de los cuidadores diseñada por el Grupo de Cuidado al Paciente Crónico de la Facultad de Enfermería de la Universidad Nacional de Colombia.

\section{Resultados}

Las características socio-demográficas del cuidador familiar de personas mayores de la Localidad de Usaquén en Bogotá D.C, Colombia, se presentan en la Tabla 1 (22), Con relación a la edad la mayoría estaba entre 36 a 59 años (53\%), mientras que el $24 \%$ estaba en el rango de 18 y 24 años y mayores de 60 años un (23\%). En cuanto al sexo del cuidador familiar, el $81 \%$ eran mujeres.

La mayoría de los cuidadores familiares sabían leer y escribir, situación que se relaciona con el nivel de escolaridad de primaria o bachillerato, donde el (45\%) cursó algún nivel, mientras que el (39\%) completó los estudios, solo el (11\%) tuvo acceso a un nivel de educación superior y el 5\% reportó no tener ningún nivel de formación.

El estado civil de los cuidadores que predominó, fue casado(a) 36\%. La ocupación de los cuidadores familiares de mayor reporte es dedicación al hogar, seguido por tener vinculación laboral y trabajo independiente. En cuanto a la seguridad social el $87 \%$ posee régimen subsidiado o sistema de salud pública. El $46 \%$ de los cuidadores familiares pertenecían al estrato 2 . 
Tabla 1. Características sociodemográficas del cuidador familiar de persona mayor (n:91)

\begin{tabular}{|c|c|c|}
\hline Variable & $\mathrm{n}: 91$ & $\%$ \\
\hline $\begin{array}{l}\text { Edad del Cuidador } \\
36 \text { a } 59 \text { años } \\
18 \text { a } 24 \text { años } \\
\text { Mayores de } 60 \text { años }\end{array}$ & $\begin{array}{l}48 \\
22 \\
21\end{array}$ & $\begin{array}{l}53 \\
24 \\
23\end{array}$ \\
\hline $\begin{array}{l}\text { Sexo del Cuidador } \\
\text { Mujeres } \\
\text { Hombres }\end{array}$ & $\begin{array}{l}74 \\
17\end{array}$ & $\begin{array}{l}81 \\
19\end{array}$ \\
\hline $\begin{array}{l}\text { ¿Sabe leer y escribir? } \\
\text { SI } \\
\text { NO }\end{array}$ & $\begin{array}{l}87 \\
4 \\
\end{array}$ & $\begin{array}{l}96 \\
4 \\
\end{array}$ \\
\hline $\begin{array}{l}\text { Nivel de escolaridad } \\
\text { Primaria y/o Bachillerato incompleto } \\
\text { Primaria y/o Bachillerato completo } \\
\text { Formación Universitaria } \\
\text { Ninguno nivel de escolaridad }\end{array}$ & $\begin{array}{l}41 \\
35 \\
10 \\
5\end{array}$ & $\begin{array}{l}45 \\
39 \\
11 \\
5\end{array}$ \\
\hline $\begin{array}{l}\text { Estado Civil } \\
\text { Casado(a) } \\
\text { Soltero(a) } \\
\text { Unión Libre } \\
\text { Separado } \\
\text { Viudo }\end{array}$ & $\begin{array}{l}33 \\
26 \\
20 \\
7 \\
5\end{array}$ & $\begin{array}{l}36 \\
29 \\
22 \\
8 \\
5\end{array}$ \\
\hline $\begin{array}{l}\text { Ocupación } \\
\text { Hogar } \\
\text { Empleado(a) } \\
\text { Trabajo Independiente } \\
\text { Dedicarse al hogar y trabajo independiente } \\
\text { Otras ocupaciones }\end{array}$ & $\begin{array}{l}48 \\
20 \\
15 \\
4 \\
4\end{array}$ & $\begin{array}{l}53 \\
22 \\
17 \\
4 \\
4\end{array}$ \\
\hline $\begin{array}{l}\text { Seguridad social } \\
\text { Régimen subsidiado } \\
\text { Régimen contributivo } \\
\text { Vinculado }\end{array}$ & $\begin{array}{l}79 \\
10 \\
2\end{array}$ & $\begin{array}{l}87 \\
11 \\
2\end{array}$ \\
\hline $\begin{array}{l}\text { Estrato socioeconómico } \\
2 \\
3 \\
1\end{array}$ & $\begin{array}{l}42 \\
25 \\
24\end{array}$ & $\begin{array}{l}46 \\
28 \\
26\end{array}$ \\
\hline $\begin{array}{l}\text { Brinda cuidado desde el diagnóstico de la Persona con ECNT } \\
\text { SI } \\
\text { NO }\end{array}$ & $\begin{array}{l}81 \\
10 \\
\end{array}$ & $\begin{array}{l}89 \\
11\end{array}$ \\
\hline $\begin{array}{l}\text { Tiempo que lleva como Cuidador } \\
\text { Más de } 37 \text { meses } \\
19 \text { a } 36 \text { meses } \\
7 \text { a } 18 \text { meses }\end{array}$ & $\begin{array}{l}79 \\
8 \\
4\end{array}$ & $\begin{array}{l}87 \\
9 \\
4\end{array}$ \\
\hline $\begin{array}{l}\text { Número de horas que dedica diariamente al Cuidado } \\
24 \text { horas } \\
\text { Menos de } 6 \text { horas } \\
7 \text { a } 12 \text { horas } \\
\text { 13 a } 23 \text { horas }\end{array}$ & $\begin{array}{l}49 \\
19 \\
14 \\
9\end{array}$ & $\begin{array}{l}54 \\
21 \\
15 \\
10\end{array}$ \\
\hline $\begin{array}{l}\text { Único Cuidador } \\
\text { NO } \\
\text { SI }\end{array}$ & $\begin{array}{l}53 \\
38 \\
\end{array}$ & $\begin{array}{l}58 \\
42 \\
\end{array}$ \\
\hline $\begin{array}{l}\text { Relación con la persona que cuida } \\
\text { Hijo(a) } \\
\text { Cuñado(a) nuera, yerno, sobrino, primo(a) } \\
\text { Esposo(a) } \\
\text { Abuelo(a) } \\
\text { Amigo(a) } \\
\text { Madre/Padre }\end{array}$ & $\begin{array}{l}49 \\
19 \\
12 \\
7 \\
3 \\
1\end{array}$ & $\begin{array}{l}54 \\
21 \\
13 \\
8 \\
3 \\
1\end{array}$ \\
\hline
\end{tabular}

Fuente: Encuesta de Caracterización de los cuidadores familiares de personas con enfermedad Crónica aplicada a cuidadores localidad de Usaquén Bogotá D.C.-Colombia, noviembre 2010-enero 2011. 
Con referencia a si el cuidador brindaba cuidado desde el momento del diagnóstico el $89 \%$ si lo hacía, refiriendo que el tiempo en dicha actividad lo realizan desde hace más de 37 meses; con una dedicación diaria de 24 horas (54\%), menos de 6 horas (21\%), entre 7 a 12 horas $(15 \%)$ y de 13 a 23 horas (10\%).

E1 58\% de los cuidadores contaba con redes de apoyo como subsidio económico, talleres para cuidadores y la vinculación de otros miembros de la familia; en algunos casos eran apoyados por cuidadores formales en la modalidad de técnicos en auxiliares de enfermería; mientras que el $42 \%$ no contaba con redes de apoyo y era único cuidador.

En cuanto a la relación de la persona mayor con el cuidador, la mayoría corresponde a hijos (as) que cuidan a sus padres, seguido por otros como cuñados (as), las nueras, yernos, sobrinos, primos (as), esposo (a), abuelo (a), un amigo (a), y en menor número madre/padre.

\section{Discusión}

En estudios similares de cuidadores familiares la edad de la mayoría de los cuidadores se encuentra en el rango de 36 a 59 años $(12,15,16,23)$. Este rango de edad se caracteriza por la multiplicidad de roles sociales: padres, hijos, esposos, trabajadores que en un momento dado puede ser causal de sobrecarga en el cuidador, porque todos exigen dedicación y esfuerzo $(9,12,15)$.

En cuanto al sexo del cuidador familiar la mayoría de los estudios mostraban similitud con los resultados de esta investigación, predominio mujeres en el rol del cuidador $(8$, 9,12-17), las mujeres como depositarias y transmisoras de conocimientos para el cuidado de la salud en el núcleo familiar. Así es como las hijas son las que asumen en su mayoría la acción de cuidar, siendo este rol una herencia cultural que ha recibido la mujer en su papel de cuidar, ya que desde edades tempranas son entrenadas para el cuidado de los hijos, por eso asumen socialmente la tarea de cuidar $(7,9)$.

El nivel educativo del cuidador corresponde con otros estudios donde la mayoría sabe leer y escribir, transitan desde algún grado de escolaridad básica $(8,12,13,15,21)$, inclusive el Universitario (15). La escolaridad es un factor protector porque proporciona elementos básicos que facilitan el fortalecimiento de la habilidad de cuidado: atención, vigilancia y acompañamiento; lo que disminuye los impactos sociales, económicos y emocionales del cuidado, además contribuye con la estructura adecuada de su red de apoyo(12).

La red de apoyo inmediata está relacionada con el estado civil de los cuidadores, para este estudio la mayoría de cuidadores son casados con un núcleo familiar conformado. La literatura reporta que los cuidadores son casado(a)s, soltero(a)s y en unión libre $(7,12,15,21)$, siendo la pareja y la familia extensa el principal apoyo en su rol como cuidador, que acompaña las responsabilidades asumidas (16); en algunos casos también se cuenta con orientación técnica de cuidadores formales a través de ayuda institucional, económica y socio-municipal; sin embargo, estos esfuerzos gubernamentales y no gubernamentales son insuficientes al compararlos con los desafíos que afrontan diariamente los cuidadores $(15,16,21)$.

En este sentido las organizaciones han centrado esfuerzos por facilitar espacios sociales de distracción que disminuyen el riesgo de sobrecarga del cuidador y potencian los mecanismos de afrontamiento (16). Se plantea la necesidad del diseño de programas tendientes a fortalecer las redes de apoyo identificadas como vitales dentro del escenario de cuidado.

La red de apoyo puede facilitar la ocupación de los cuidadores familiares, el estado civil soltero-a con red de apoyo identificado, permite y da la oportunidad de asumir ocupaciones diferentes a la del hogar, aunque prevalece la dedicación exclusiva al cuidado como se muestra en otros estudios (7). Sin embargo, aunque el vínculo laboral no es estable, predomina el trabajo informal (por horas, y actividades) que les ayuda a suplir las necesidades económicas teniendo en cuenta que la mayoría pertenece a estrato 2 y 3 y el nivel educativo y el tiempo que dedican al cuidado no les permite adquirir mayores compromisos laborales. Se considera que el mantener un rol laboral tiene varios efectos: en primer lugar, puede ser un factor protector que disminuye las repercusiones del cuidado sobre la salud del cuidador, como ocurre en la persona que es ama de casa, lo que exige una alta dedicación al cuidado, generando restricciones de la vida social, teniendo consecuencias sobre su desarrollo personal, la autoestima y de su red de apoyo (13). En segundo lugar, puede ser un factor causal de sobrecarga de roles, generador de angustia y estrés por las necesidades económicas que tiene el hogar y por las corresponsabilidades de cuidado adquiridas que disminuyen el tiempo de dedicación en el mercado laboral.

Para este estudio la actividad principal de los cuidadores familiares de personas mayores es el hogar $(8,12,15)$, otras actividades identificadas como ser empleado(a), o ser trabajador independiente. A pesar que la ocupación de los cuidadores de este estudio no es exclusiva al cuidado de la persona mayor, la mayoría inicia el cuidado desde el momento del diagnóstico $(12,24)$, donde el reconocimiento y aceptación de la enfermedad provoca alteraciones no solo en el enfermo sino también en la familia, su aparición produce una redistribución de los roles familiares y genera iniciativas de atención y acompañamiento en las actividades diarias de la persona mayor con el propósito de darle bienestar(7).

La mayoría de los cuidadores brindan apoyo y soporte a sus familiares por largos periodos, situación que es coincidente 
con la de este estudio; los cuidadores han ejercido su labor por más de 37 meses, en muchos de los casos los cuidadores brindan acompañamiento por 24 horas (12), porque su ocupación como amas de casa, les permite permanecer en el hogar $(7,8)$, algunas de ellas tienen vínculos laborales informales gracias a que cuentan con una red de apoyo para el cuidado. Algunos autores describen que los cuidadores cuentan con poca o nula red de apoyo por lo que deben brindar cuidado durante 24 horas, lo que eleva los niveles de depresión y angustia por la incertidumbre que genera el estado de salud de la persona mayor, acelerando la aparición de problemas de salud física: alteraciones cardiovasculares e inmunológicos, además de estados prolongados de estrés $(13,22,25)$.

Las intervenciones de enfermería asociadas a las condiciones socioeconómicas del cuidador familiar debe estar orientadas al reconocimiento de los cuidadores como actores esenciales del sistema de salud, facilitar "herramientas educativas" $(22,26)$, para mejorar la habilidad de cuidado, de tal manera que logren satisfacer las necesidades de la persona enferma, además que den respuesta a la problemática familiar, y para mantener la propia salud y fomentar una buena relación con la persona cuidada $(17,22,27-29)$.

Escuredo-Rodríguez (30), considera la necesidad de promover y apoyar a los Grupos de Ayuda Mutua (GAM), como un sistema de soporte que permita al cuidador intercambiar trucos y experiencias, formas de producción en el hogar, mecanismos de fortalecimiento de vínculos y dinámicas familiares que mejoren los sistemas de comunicación, de expresión, de afrontamiento y de resolución de conflictos, lo que puede orientar la toma de decisiones de manera individual y familiar.

Algunos autores proponen la búsqueda de alternativas de apoyo a estos cuidadores que no poseen herramientas suficientes como programas de ayuda mutua o autoayuda a cuidadores, intervenciones educativas para enfrentar las situaciones negativas derivadas del cuidado como afectación de su salud física, psicológica(31).

\section{Conclusiones}

El cuidador familiar de la persona mayor se caracteriza por ser de sexo femenino, hijo(a) de la persona mayor, tiene un nivel de escolaridad de primaria incompleta, casado(a), su principal ocupación es el hogar. Con predominio de la afiliación al sistema público de salud, ligada a los estratos 1 , 2 y 3 . Ellos cuidaron a la persona mayor desde el momento del diagnóstico, por más de 37 meses, dedicaban diariamente a esta labor 24 horas; algunos contaban con redes de apoyo identificadas: la familia y los talleres ofertados por el proyecto 496 "años dorados" de la Localidad de Usaquén Bogotá-Colombia.
Son razones afectivas las que impulsan a ofrecer los cuidados a pesar de no contar con experiencia y entrenamiento previo. Se presentan afectaciones múltiples relacionadas con la salud física y mental, así como en el ámbito social y económico, por lo que se hace inminente la búsqueda de alternativas de apoyo para los cuidadores que no cuentan con recursos para acceder a niveles óptimos de información acerca de las enfermedades crónicas que presenta el adulto mayor, al que ofrecen sus cuidados (11).

Para el profesional de enfermería el estudio de las características socio-demográficas le permite explorar el cuidado en otros contextos, que involucran dimensiones, relaciones, factores y vínculos que se convierten en retos frente al diseño, ejecución y evaluación de intervenciones de apoyo como respuesta a las necesidades identificadas, para afrontar los conflictos, miedos, desafíos que experimente la población (22). Este estudio hace evidente la realidad de los cuidadores, de personas mayores con enfermedad crónica no trasmisible.

\section{Conflicto de intereses}

Las autoras manifiestan no tener conflicto de intereses.

\section{Agradecimientos}

A la Universidad de Ciencias Aplicadas y Ambientales (UDCA) por facilitar los recursos y brindar el apoyo para el desarrollo del proyecto de investigación. A la Secretaría de Integración Social de Usaquén BogotáColombia, por el apoyo a través de su equipo de trabajo y de programas con iniciativa social que fomentan el desarrollo de procesos de investigación.

\section{Referencias}

1. Berrío MI. Envejecimiento de la población: un reto para la salud pública. Rev Colomb anestesiol. 2012; 40(3): 192-194.

2. Cardona D, Peláez E. Envejecimiento poblacional en el siglo XXI: oportunidades, retos y preocupaciones. Salud Uninorte. Barranquilla (Col.) 2012; 28(2):335-348.

3. Serra MA. Las enfermedades crónicas no transmisibles en la Convención Internacional Cuba-Salud 2015. Revista Finlay [revista en internet]. 2015 [Acceso 10 dic 2015]; 5(2): [aprox. 2 p.]. Disponible en: http://www. revfinlay.sld.cu/index.php/finlay/article/view/362.

4. Brenes L, Menéndez J, Guevara A. "Comorbilidad y Discapacidad: su relación en Adultos Mayores de Ciudad de La Habana". GEROINFO. RNPS. 2006; 2(2):1-24. Disponible en: http://repositoriocdpd.net:8080/bitstream/ handle/123456789/367/Art BrenesHern\%C3\%A1ndez L_Comorbilidad Discapacidad_2006.pdf?sequence=1.

5. González A. La autonomía del paciente con enfermedades crónicas: De paciente pasivo a paciente activo. Enferm Clin. 2014; 24(1):67-73. 
6. Silva E, Rodríguez J, Zas V. "Promoción de salud y envejecimiento activo". Geroinfo. 2013; 8(1):1-15.

7. Zanetti M. Las enfermedades crónicas no transmisibles y tecnologías en salud. Rev. Latino-Am. Enfermagem [revista en internet]. 2011 [Acceso 21 Dic 2014]; 19(3):12. Disponible en: http://www.scielo.br/pdf/rlae/v19n3/ es_01.pdf.

8. Carrillo GM, Sanchéz B, Barrera L. Habilidad de cuidado de cuidadores familiares de niños y adultos con enfermedad crónica. Index Enferm [revista en internet]. 2014 [Acceso 16 Dic 2015]; 23(3):129-133. Disponible en: http://scielo.isciii.es/scielo.php?script= sci_arttext\&pid=S1132-12962014000200003\&Ing=es. http://dx.doi.org/10.4321/S1132-12962014000200003.

9. Ferraz K, Cruz V, Ramon J, Silva R, Nagib E, Missias R. Calidad de vida de cuidadores familiares de ancianos: una revisión integradora. Revista Cubana de Enfermería [revista en internet]. 2013; 29(4):1-6. Disponible en: http://www.revenfermeria.sld.cu/index.php/enf/article/vie w/253/73.

10. Galvéz M, Saucedo MC, Calderón A, Pacheco I, Carmona F, García AM. El proceso de afrontamiento en cuidadoras familiares de personas con alto grado de dependencia. Distrito Sanitario Costa del Sol, Servicio Andaluz de Salud (SAS). Fundación Index. 2014; 20(20):1-5 [Documento en Internet]. Disponible en: http://www.index-f.com/para/n20/pdf/374.pdf.

11. Ocampo J, Herrera J, Torres P, Rodríguez J, Loboa L, García C. Sobrecarga asociada con el cuidado de ancianos dependientes. Colomb Med 2007; 38:40-46.

12. Carrillo, G, Chaparro L, Sánchez B. Carga del cuidado en cuidadores familiares de personas con enfermedad crónica en la región pacífica colombiana. Cienc. enferm. [Online]. 2014; 20(2): 83-91.

13. Lund L, Ross L, Aagaard M, Groenvold M. Cancer caregiving tasks and consequences and their associations with caregiver status and the caregiver's relationship to the patient: a survey. BMC Cáncer. 2014; 14(541):1-13.

14. Ganapathy $\mathrm{V}$, et al. Caregiver burden, productivity loss, and indirect costs associated with caring for patients with poststroke spasticity. Clinical Interventions in Aging 2015:10 1793-1802.

15. Úbeda I. Calidad de vida de los cuidadores Familiares: evaluación mediante un cuestionario. Universidad de Barcelona. [Tesis]. 2009 [Acceso 2013 Mar 20]; 1-293. Disponible en: http://www.tdx.cat/bitstream/handle/ 10803/2081/IUB TESIS.pdf;jsessionid=C2099CEBC3 E875CD1F6F781B30CF6D29.tdx2? sequence $=1$.

16. Vaquiro S, Stiepovich J. Cuidado informal, un reto asumido por la mujer. Ciencias y enfermería [revista en la internet]. 2010 [Acceso 20 Mar 2013]; 16(2):9-16. Disponible en: http://www.scielo.cl/pdf/cienf/v16n2/ art 02.pdf

17. Castaño C, Perea D, Hurtado L, Sánchez A. Calidad de vida relacionada con la salud de los pacientes y sus cuidadores en una unidad renal de la ciudad de Cali en 2013. Ciencia \& Salud. 2014; 2(8):29-35.

18. Cerquera A, Granados F, Buitrago A. Sobrecarga en cuidadores de pacientes con demencia tipo alzheimer. Psychol. av. discip. 2012; 6(1): 35-45.

19. Ganapathy V, et al. Caregiver burden, productivity loss, and indirect costs associated with caring for patients with poststroke spasticity. Clinical Interventions in Aging 2015:10 1793-1802.

20. República de Colombia. Ley 911 de 2004: Código Deontológico de Enfermería. 2014 [Acceso 27 Mar 2013]; 23 p. Disponible en: http://nuevo.unisystem.edu.co/ wpcontent/uploads/2014/10/ley 911 de 2004.pdf.

21. República de Colombia. Resolución $\overline{\mathrm{N}}^{\circ} 008430$ de 1993. Ministerio de Salud. [Documento en internet]. 1993. [Acceso 27 Mar 2014]: 1-12. Disponible en: http://www. unisabana.edu.co/fileadmin/Documentos/Investigacion/ comite_de_etica/Res_8430_1993___Salud.pdf

22. Encuesta de Caracterización de los cuidadores familiares de personas con enfermedad Crónica aplicada a cuidadores localidad de Usaquén Bogotá D.C.Colombia, Noviembre 2010-enero 2011.

23. Delgado E, Suarez O, De Dios R, Valdespino I, Sousa Y, Braña G. Características y factores relacionados con sobrecarga en una muestra de cuidadores principales de pacientes ancianos con demencia. Semergen. 2014; 40(2):57-64.

24. LeSeure P, Chongkham S. The Experience of Caregivers Living with Cancer Patients: A Systematic Review and Meta-Synthesis. J. Pers. Med. 2015; 1(5): 406-439.

25. Flórez I, Montalvo A, Herrera A, et al. Afectación de los bienestares en cuidadores de niños y adultos con enfermedad crónica. Rev. salud públic [revista en internet]. 2010 [Acceso 07 Oct 2014]; 12(5):754-764. Disponible en: http://www.scielo.org.co/scielo.php? script=sci_ arttext\&pid=S0124-00642010000500006\&lng=en.

26. Megret A, Naranjo M, Fong Y. Educación a familiares sobre el manejo del adulto mayor dependiente. Rev Cubana Enfermer [revista en la Internet]. 2002[Acceso 28 Mar 2013]; 18(1):43-49. Disponible en: http://scielo. sld.cu/scielo.php?script=sci_arttext\&pid=S086403192002000100008\&lng=es.

27. Montalvo A, Bradán Y, Cavadias C, Medina E, Méndez K, Padilla C, et al. Habilidad de cuidado de cuidadores familiares principales de pacientes con ACV. Cartagena (Colombia). Salud Uninorte [revista en internet]. 2010 [Acceso 27 Mar 2013]; 26(2):1-6. Disponible en: http://rcientificas.uninorte.edu.co/index.php/salud/article /view/200/5806.

28. Ávila J, García J, Gaitán J. Habilidades para el cuidado y depresión en cuidadores de pacientes con demencia. revista colombiana de psicología. 2010; 19(1):71-84.

29. Mediavilla M, Rodríguez A, Ocaña MA, Baquera MJ, Morel S. Programa de educación a cuidadores de pacientes dependientes hospitalizados para mejorar la calidad de vida. NURE Inv [revista en internet]. 2014; 11(68): [aprox. 15 p.]. Disponible en: http:// www.fuden.es/FICHEROS ADMINISTRADOR/PROY ECTO/NURE68_proyecto_educacion.pdf

30. Escuredo-Rodriguez B. El discurso de las enfermeras ante el cuidado de las personas mayores dependientes y sus cuidadores familiares. Index de enfermería. [revista en internet]. 2006 Mar [Acceso 28 mar 2013]; 15(52-53): 1-9. Disponible en: http://www.index-f.com/indexenfermeria/52-53/r4548.php.

31. Losada A, et al. Atendiendo a las variadas problemáticas de los cuidadores familiares de personas con demencia: aportaciones de la terapia cognitivo-conductual y de la terapia de aceptación y compromiso. Clínica y Salud. 2015; 26(1):41-48. 